Review

\title{
Induction of Chromosomal Instability via Telomere Dysfunction and Epigenetic Alterations in Myeloid Neoplasia
}

\author{
Beate Vajen $^{\dagger}$, Kathrin Thomay ${ }^{\dagger}$ and Brigitte Schlegelberger * \\ Institute of Cell and Molecular Pathology, Hannover Medical School, Carl-Neuberg-Str. 1, \\ 30625 Hannover, Germany; E-Mails: vajen.beate@mh-hannover.de (B.V.); \\ thomay.kathrin@mh-hannover.de (K.T.)
}

$\dagger$ These authors contributed equally to this work.

* Author to whom correspondence should be addressed; E-Mail: schlegelberger.brigitte@mhhannover.de; Tel.: +49-511-532-4522; Fax: +49-511-532-4521.

Received: 17 April 2013; in revised form: 17 June 2013 / Accepted: 25 June 2013 /

Published: 4 July 2013

\begin{abstract}
Chromosomal instability (CIN) is a characteristic feature of cancer. In this review, we concentrate on mechanisms leading to $\mathrm{CIN}$ in myeloid neoplasia, i.e., myelodysplastic syndrome (MDS) or acute myeloid leukemia (AML). The pathogenesis of myeloid neoplasia is complex and involves genetic and epigenetic alterations. Chromosome aberrations define specific subgroups and guide clinical decisions. Genomic instability may play an essential role in leukemogenesis by promoting the accumulation of genetic lesions responsible for clonal evolution. Indeed, disease progression is often driven by clonal evolution into complex karyotypes. Earlier studies have shown an association between telomere shortening and advanced MDS and underlined the important role of dysfunctional telomeres in the development of genetic instability and cancer. Several studies link chromosome rearrangements and aberrant DNA and histone methylation. Genes implicated in epigenetic control, like DNMT3A, ASXL1, EZH2 and TET2, have been discovered to be mutated in MDS. Moreover, gene-specific hypermethylation correlates highly significantly with the risk score according to the International Prognostic Scoring System. In AML, methylation profiling also revealed clustering dependent on the genetic status. Clearly, genetic instability and clonal evolution are driving forces for leukemic transformation. Understanding the mechanisms inducing CIN will be important for prevention and for novel approaches towards therapeutic interventions.
\end{abstract}


Keywords: chromosomal instability; myeloid leukemia; MDS; AML; telomeres; epigenetics; genetic instability

\section{Introduction}

\subsection{Chromosomal Instability in Cancer}

Maintenance of genomic integrity is of the utmost importance for an organism's survival and a prerequisite for successfully passing on genetic information. However, the stability of the genome is constantly threatened by DNA replication errors and by DNA damage due to a multitude of exogenous and endogenous factors, e.g., oxidative stress. The cell continuously counteracts these processes and has developed a complex network to repair genetic alterations. Nonetheless, a small number of cells may not succeed in repairing damaged DNA. In this situation, the cell usually enters apoptosis. However, in some cases mutations, like in oncogenes or tumor suppressor genes, may occur that allow the cell to bypass apoptosis and to survive. If such a cell acquires additional genetic alterations providing a clonal advantage, this may lead to malignant transformation and ultimately to cancer [1].

Genomic instability may result in numerous defects like replication errors, telomere dysfunction, epigenetic changes or defective DNA repair as a few examples. Chromosomal instability (CIN), a form of genomic instability, is by definition the cell-to-cell variability with regard to chromosomal changes, e.g., the rate of gross chromosome changes like an imbalance in the number of chromosomes per cell and an enhanced rate of loss of heterozygosity [2-4]. As such, chromosomal instability may predispose to the outgrowth of clones containing "fixed" chromosome aberrations. Chromosome aberrations in myeloid neoplasms include whole-chromosome aberrations like monosomy or trisomy or structural changes like deletions, translocations and inversions [5]. Therefore, Bajani et al. [6] suggest to distinguish between structural chromosomal instability (S-CIN), developed at unstable genomic regions, or through aberrant DNA repair or methylation and numerical CIN (N-CIN), which occur due to mitotic segregation errors. Chromosomal instability can be found in all kinds of human cancer and is usually a strong negative prognostic indicator $[7,8]$. Also in myelodysplastic syndromes, elevated chromosomal instability, i.e., the cell-to-cell variability as determined by fluorescence in situ hybridization, correlates with poor outcome, irrespective of the cytogenetic subtype [9].

\subsection{Role of Chromosomal Aberrations in Human Leukemia}

In this review we discuss the role of chromosomal and genomic instability in myeloid neoplasia, like myelodysplastic syndrome (MDS) and acute myeloid leukemia (AML). MDS are a heterogeneous group of malignant clonal disorders of hematopoietic stem cells, characterized by dysplastic cells and an ineffective hematopoiesis in one or more myeloid cell lineages [10]. According to the World Health Organization (WHO), MDS is subdivided into different morphological subtypes. The International Prognostic Scoring System (IPSS) categorizes these subtypes into different risk groups (low, intermediate 1 and 2 and high) based on the number of cytopenias, the karyotype, and the percentage of blasts in the bone marrow [10,11]. Generally, in MDS, the amount of blasts, the immature 
hematopoietic progenitor cells, in the bone marrow is less than $20 \%$. This definition distinguishes it from AML with an amount of blasts of more than $20 \%$ [12]. In about $10 \%$ to $50 \%$ of cases, strongly depending on the respective subtype, myelodysplastic syndromes show a propensity to progression into AML $[13,14]$.

In MDS, clonal chromosome aberrations have important diagnostic, prognostic and clinical relevance $[10,15]$. In a high amount of cases, MDS shows progression into AML, which is often associated with karyotypic evolution, i.e., the acquisition of additional chromosomal aberrations.

In general, clonal chromosome aberrations can be found in about half of the AML and MDS patients [16]. MDS shows mainly unbalanced aberrations [17]. The most common chromosomal aberrations in MDS are monosomy 7, loss of the $\mathrm{Y}$ chromosome, trisomies 8 and 21 and deletions in $5 q, 7 q, 17 p$ and 20q. Losses of 5q, 7q and 17p are also frequent aberrations of complex karyotypes, which by definition contain three or more clonal aberrations.

Cytogenetic abnormalities in AML and MDS are associated with distinct clinical and morphological subtypes and often predict disease outcome. For example, MDS with an isolated del(5q) defines a subtype of MDS with a favorable outcome, a median survival of 77.2 months, and a low risk of transformation into AML. In contrast, complex karyotypes are associated with a very high risk of transformation into AML and with a very poor prognosis, i.e., a median survival of 8.8 months [10]. Since complex karyotypes frequently occur in patients with secondary MDS, who have been treated with combined chemo- and radiotherapy due to a primary malignancy, increased genomic instability may lead to the development of aberrant clones.

As MDS typically shows unbalanced aberrations like deletions and monosomies, it can be concluded that the molecular mechanism in MDS is predominantly loss or inactivation of a tumor suppressor gene, in contrast to AML, where often balanced translocations and inversions occur that may induce proliferation or inhibit differentiation [16,18]. As examples, a translocation $\mathrm{t}(8 ; 21) / A M L 1-E T O$ fusion and an inv(16)/CBFb-MYH11 fusion result in the inactivation of core binding factors that have an essential role in myeloid differentiation. Both AML subtypes are associated with a good prognosis [12]. A $\mathrm{t}(15 ; 17) / P M L-R A R A$ fusion resulting in the inactivation of the retinoid acid receptor $\alpha$ also defines an AML subtype with good prognosis. The molecular defect can be counteracted by treatment with retinoic acid, which induces the differentiation of the myeloid blasts [19]. Inversion of 3q, translocations of $M L L$, a t $(6 ; 9) / D E K-N U P 214$ fusion or a complex aberrant karyotype are each associated with a poor prognosis [12,20,21].

Genes frequently found to be mutated in karyotypically normal AML are NPM1, FLT-3 and CEBPalpha [22]. Recently, recurrent mutations with prognostic significance have been discovered in MDS, among those mutations in the genes TP53, EZH2, ETV6, GNAS, RUNX1, and ASXL1 [23].

\subsection{Mechanisms to Induce CIN in Myeloid Neoplasia}

The pathogenesis of myeloid neoplasia is generally complex and involves genetic, epigenetic and immune-mediated mechanisms [11]. As in many cancer types, chromosomal instability and clonal evolution play an essential role in leukemogenesis by promoting the accumulation of genetic lesions responsible for malignant transformation. Although exogenous factors like previous chemo- and 
radiotherapy increase the risk to develop MDS and AML, and particularly those with complex karyotypes, the exact mechanisms inducing CIN are not clear.

Aberrations specific for MDS and AML, particularly terminal deletions, unbalanced translocations as well as gains and losses, could theoretically result from dysfunctional telomeres [24]. Earlier studies have shown an association between shorter telomeres and advanced MDS [25,26]. Also in AML, an increasing number of aberrations seem to be associated with ongoing telomere erosion, resulting in critically short telomeres of one or more chromosomes [24]. Data from animal models and in vitro experiments have elucidated an important role of dysfunctional telomeres in the development of genetic instability and cancer, particularly MDS and AML [24,27]. Recent studies not only underlined the significance of the mean telomere length, but particularly of single or very few critically short, dysfunctional telomeres causing chromosomal instability $[28,29]$. In the clinical context, however, the relevance of critically short telomeres for the development of CIN in MDS and AML remains to be shown.

The identification of recurrent mutations in genes involved in the epigenetic regulation in patients with MDS has led to new insights into the pathophysiology of this disorder. Of particular interest is the recent recognition of mutations in genes involved in histone modification (EZH2 and $A S X L 1$ ) and DNA methylation (DNMT3A and TET2). For time-controlled activation and silencing of tissue-specific genes in eukaryotic cells, continuous remodeling of the chromatin structure is necessary. Epigenetic factors modify the DNA and DNA-associated histones, thereby inducing conformational changes of the chromatin that allow activation or repression of gene expression. Mutations of epigenetic modifiers provide an important link between genetic and epigenetic alterations in MDS. In AML, gene expression studies also observed differential expression of the DNMT3A and DNMT3B genes, coding for DNA methyltransferases [30]. Furthermore, aberrant DNA methylation patterns helped to identify new subgroups of AML. Notably, cytogenetic subgroups are characterized by distinct DNA methylation patterns. Particularly for AML with normal karyotype, they define a methylation-based outcome predictor for disease-free and overall survival [31,32].

Recently, the important role of histone methylation for the induction of chromosomal and genomic instability and leukemia pathology in humans and mice became evident. Of course, it is not only telomeric dysfunction or epigenetic alterations that play an important role in the induction of chromosomal and genomic instability in myeloid malignancies. Chromosome aberrations have, for example, also been described to occur due to defective cytokinesis or cell cycle regulation [33,34]. Also, increased DNA damage, either via internal mechanisms leading to increased reactive oxygen species (ROS) or external factors like chemicals, cancer therapy or radiation, or a defective DNA damage repair in general due to mutations, may lead to aberrations in myeloid leukemia [35-37].

Yet, the mechanisms by which epigenetic alterations and telomere dysfunction contribute to disease pathogenesis are a highly active area of research and therefore we would like to address the following question in this review: How can telomeres and epigenetics contribute to inducing chromosomal and genomic instability and what role does this play in leukemogenesis? 


\section{Telomeres}

\subsection{Telomeres and CIN}

Progressive telomere shortening due to the end-replication problem is one of the molecular mechanisms underlying aging. Starting at a length of around $15 \mathrm{~kb}$ and with an approximate telomere loss of 30-150 bp with each cell division, continued telomere length shortening can be observed over time. However, within the first two years of life, a more rapid loss of 1,000-3,000 bp/year takes place. Furthermore, accelerated telomere shortening can also be observed after the age of 60 years. The reason for this might, for example, lie in replicative stress due to a diminishing hematopoietic stem cell pool [38]. Yet, excessive telomere shortening also occurs during excessive proliferation after stem cell depletion or in cancer cells.

As already mentioned, short telomeres, e.g., due to excessive proliferation, induce senescence when they reach a critical length of about 5-8 kb, the so-called Hayflick limit [39]. Importantly, single critically short telomeres are sufficient to elicit this cellular response. This finding demonstrates that, besides the median telomere length of the cell, the length of the single chromosome arms is also of great importance [40].

Dysfunctional telomeres lead to senescence and thus limit the proliferative potential of a stem cell. This might possibly lead to selection of stem cells with a defective DNA damage response, in which no cell-cycle arrest occurs due to deficient or low repair capacity and which are therefore prone to chromosomal instability predisposing them for leukemia [41].

Usually, cells that are able to circumvent senescence enter a further growth arrest phase (crisis), in which critically short telomeres of about 3-4 kb tend to fuse with other "free ends" and thus promote further genomic instability [42]. Elimination of cells with short telomeres due to either aging or excessive proliferation is thus an important tumor-suppressive mechanism of the cell and a hurdle to tumor progression $[43,44]$.

However, in the case of further mutations as in TP53 and $R B$, cells may be able to bypass senescence and crisis. In the case of continued proliferation, the cells with short telomeres might turn into immortalized cancer stem cells, in which chromosomal aberrations are stabilized by up-regulation of telomerase or by induction of ALT [45,46] (Figure 1). In line with this, in MDS a complex karyotype is observed more often in older patients, naturally with a background of shorter telomeres, than in younger patients, pointing towards short telomeres as a risk factor for chromosomal instability.

Cancer stem cells generally show genetic aberrations, telomere stability and telomerase activity, suggesting that these cells have initially gone through a phase with very short telomeres, which have then at a later stage been re-elongated and stabilized [42,47].The appearance of these telomerase-positive clones with stabilized chromosome aberrations might have evolved due to the selective growth advantage of cells with activated telomerase [44].

Thus, critically short telomeres, even single short telomeres within a cell, can induce end-to-end fusions and the formation of chromatin bridges during anaphase with subsequent breakage-fusion-bridge events (B/F/B cycles). These events can lead to either structural rearrangements due to sister chromatid fusions, extensive chromatin fragmentation, to the loss of whole chromosomes via mechanical disruption of the spindle machinery or to failure of cytokinesis leading to polyploidization and multiple spindle 
configurations [48,49]. Cycles of breakage-fusion-bridge events stop after acquisition of new telomeres by non-reciprocal translocation to the end of the unstable chromosome (telomere capture).

Figure 1. Imaging methods for chromosomal instability and shortened telomeres (a) Telomere/Centromere (T/C)-FISH metaphase of a healthy individual; (b) T/C-FISH metaphase of a patient with MDS and shortened telomeres; (c) Fluorescence R-banding karyotype of a patient with AML and complex aberrant karyotype, (d) Multicolor FISH karyotype of the same patient.

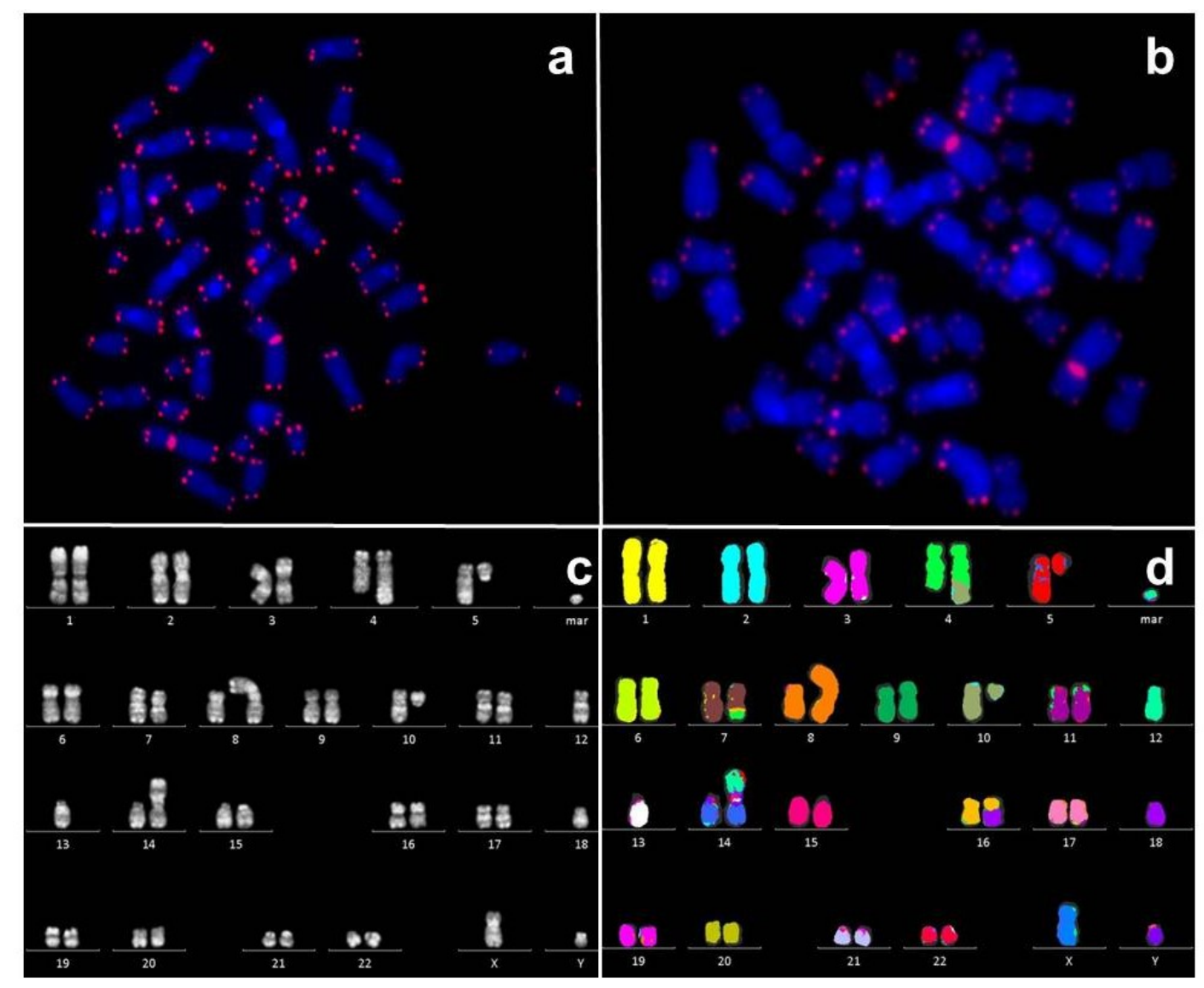

\subsection{Telomeres in Leukemia}

During the development of MDS and AML, due to excessive proliferation and/or another underlying defect, pathologically shortened telomeres in a hematopoietic stem or progenitor cell may eventually reach a critically short length. As described, this may lead to increased CIN and genetic aberrations [48]. Aberrations typical for these diseases, particularly terminal deletions, unbalanced translocations as well as gains and losses of whole chromosomes could theoretically result from dysfunctional telomeres [24].

Specifically, short telomeres may lead to the generation of dicentric chromosomes, which may induce $\mathrm{B} / \mathrm{F} / \mathrm{B}$ cycles that may subsequently lead to loss or gain of chromosomes or to the development of unbalanced changes, typical for MDS. If these chromosome aberrations provide a survival benefit or a proliferative advantage, this may then, at a later stage, lead to clonal evolution of aberrant clones and possibly to the re-activation of telomerase. Indeed, increased telomerase activity has been described in 
patients with advanced forms of MDS [50]. Also in AML, increased TERT expression, usually a characteristic feature of cancer cells, can be found in correlation with the complexity of the karyotype as well as the disease severity [51].

An association with MDS, especially with regard to the severity of the subtype and short telomeres, has already been shown $[25,26,52]$. As shown by Boultwood et al., telomere length showed a certain degree of heterogeneity within the subtypes but short telomeres could still be linked to leukemic transformation and complex aberrant karyotypes. Also, Sieglova et al. showed an association between telomere shortening and disease progression suggesting telomere dynamics as a prognostic factor in MDS. Likewise, an increasing number of aberrations in AML has been described to be associated with ongoing telomere erosion on the one hand and critically short telomeres on the other, pointing towards an essential role of telomeres in the pathogenesis of these diseases [24,53].

Recent studies not only underlined the significance of the mean telomere length, but in particular of single or very few critically short, dysfunctional telomeres causing CIN [28,29]. As an example, it has already been shown in $\mathrm{mTR}^{-/-}$mice that chromosomes frequently involved in aberrations have the shortest telomeres, leading to the conclusion that these short dysfunctional telomeres limit cellular survival [28].

Studies profiling the three-dimensional architecture of telomeres in patients with MDS and AML have shown that, based upon parameters like telomere numbers, telomere aggregates, signal intensities, nuclear volumes and nuclear telomere distribution, patients can be subdivided into distinct subgroups [54]. Notably, the evolution of telomeric dysfunction is linked to the progression of MDS into AML.

The role of telomeres, especially in MDS, becomes clear when considering diseases like dyskeratosis congenita (DC) or aplastic anemia (AA), in which a mutation in one of the telomerase components or of the "shelterin" complex (telomere-binding and stabilizing proteins) leads to critically short telomeres $(<5 \mathrm{~kb})$ and a substantially increased risk of MDS [55]. DC is a premature aging syndrome that - among other symptoms - is characterized by cytopenia in one or more lineages. This is why DC is often first diagnosed when MDS develops. Besides loss-of-function mutations in one of the telomerase components, mainly TERT, TERC and DKC, but also NOP10 and NHP2, mutations in the telomereassociated protein TINF2 have also been found in patients with DC [56,57]. These mutations lead to either telomerase deficiency (as with the X-linked $D K C 1$ ), a dysfunctional telomere repair complex (as with the autosomal dominant TERT and TERC mutations) or disrupted telomeres (as with the TINF2 mutations). Although showing differences in the clinical manifestation as well as the severity of symptoms, all these mutations lead to shortened telomeres [56].

Recently, hypomorphic mutations of TERT have been found in a subset of patients with AML that also seemed to be associated with the occurrence of specific aberrations, in particular trisomy 8 , inversion 16, a translocation $\mathrm{t}(15 ; 17)$ and a complex aberrant karyotype [41]. In aplastic anemia and MDS, somatic mutations in TERT or TERC can be observed [58]. In AA, short telomeres have directly been linked to the probability of developing a cytogenetically abnormal clone, underlining the role of telomerase and short telomeres in malignant transformation of hematopoietic stem cells and MDS development [58-60].

Intriguingly, these data provide a first hint that short telomeres contribute to the pathogenesis of MDS and AML. However, the extent to which short telomeres induce chromosomal instability and the development of aberrant clones still remains to be elucidated. It is also of high importance for patient 
management, whether the current telomere length may predict the risk to develop MDS and AML and whether a patient's telomere length is able to predict the risk of progression and relapse.

\subsection{Telomere Length Measurement in Primary Cells of Patients and Our Data}

According to our hypothesis, different cytogenetic subtypes of MDS may show different telomere length profiles. Possibly those chromosomes involved in or prone to specific aberrations may have shorter telomeres than normal chromosomes. These critically short telomeres may be a driving force towards leukemic transformation and clonal evolution. However, they might be masked in the average telomere length by longer telomeres of other chromosomes in the same cell. Therefore, determining the telomere length of each chromosome is necessary to answer this question.

To elucidate this, we recently selected a cohort of 78 patients with different cytogenetic and morphological subtypes of MDS and a cohort of 18 age and gender-matched healthy controls for telomere length measurement. In particular, we investigated the telomere length profiles and the role of single critically short telomeres by applying telomere/centromere-FISH (T/C-FISH) [61]. This method, basically a quantitative FISH in which the telomeric signal intensities are set in relation to the signal intensity of centromere 2 as an internal reference, was used in combination with fluorescence R-banding analysis of the same metaphases. This particularly allowed telomere length measurement of each single distinct chromosome arm and importantly, in the case of patients with an aberrant karyotype, guaranteed measurement of the aberrant clone excluding falsification of the results by normal cells. Bone marrow cells of patients with MDS showed significantly shorter telomeres than those of healthy controls. However, no association between short telomeres and specific cytogenetic or morphological subtypes was found and telomere lengths did not differ significantly between distinct morphological subtypes of MDS. Yet, so-called neo-telomeres (telomeres at the fusion site of two chromosomes) were found in two patients with a complex karyotype, showing a role of short telomeres in disease pathogenesis. We therefore hypothesized that, in most patients with MDS and monosomy 7 or complex karyotype, formerly short telomeres are stabilized or elongated by reactivation of telomerase or the ALT mechanism. Here, aberrant cells may have gone through a phase of very short telomeres, but survived crisis and evolved to clones after telomere-elongating mechanisms were triggered. Correspondingly, patients with a less severe subtype and mild telomere shortening might not show significant differences compared to patients with advanced MDS, whose telomeres have probably at an earlier stage gone through a phase of excessive shortening, but have already been re-elongated.

Thus, it became clear that the different cohorts were too heterogeneous, as the disease status and the time point after diagnosis have to be taken into account. To gain more information, we subsequently performed follow-up analyses in patients with MDS with an isolated deletion of 5q [62]. At an early time point after initial diagnosis and prior to lenalidomide treatment, patients who had a later disease progression showed significantly shorter telomeres than patients without progression. At the time point of progression, all patients showed a re-elongation of the median telomere length that was similar to that measured in patients without progression. Thus, at an early time point after diagnosis, short telomere length seems to indicate an increased risk of relapse and disease progression, and with this provides further evidence of telomere shortening playing a fundamental role in the malignant transformation of hematopoietic stem cells. In general, the actual telomere length has to be interpreted 
carefully, depending on different factors such as disease state and treatment. In MDS, it is not yet clear whether telomere shortening is a causative factor, as in dyskeratosis congenita, or whether the increased proliferation of MDS cells concomitantly leads to telomere shortening. On the practical side, measurement of telomere length in patients with MDS and AML could therefore be a tool for selecting those patients who might, in the first step, be monitored more closely to recognize disease progression at an early stage. Thus, telomeres not only play a role in disease pathogenesis and progression, but they may also serve as an important tool for risk assessment and therapeutic decisions.

\section{Epigenetics}

\subsection{Mutations of Epigenetic Regulators}

Somatic mutations in epigenetic regulators are a common genetic event in myeloid neoplasia and contribute to hematopoietic transformation. Epigenetic regulators can change the gene expression without changing the nucleotide sequence. Genomic DNA is wrapped around the histone proteins $(\mathrm{H} 2 \mathrm{~A}, \mathrm{H} 2 \mathrm{~B}$, $\mathrm{H} 3$ and H4), whose N-termini can be modified, constituting the chromatin. These modifications include acetylation, methylation, ubiquitylation, phosphorylation and SUMOylation. A strong interaction between the genomic DNA and the histones results in a compact chromatin formation, whereas weak interactions enable gene expression by an open chromatin structure. Analyses of recurring translocations like $\mathrm{t}(11 ; 16)(\mathrm{q} 23 ; \mathrm{p} 13.3)$, documented in cases of secondary AML or MDS, revealed the epigenetic translocation partners MLL (histone methyltransferase) and CBP (histone acetyltransferase) for the first time [63]. Up to now, many epigenetic modifiers are shown to be involved in translocations like MOZ [64], a histone lysine acetyltransferase or NUP98, which provide evidence of the importance of deregulated epigenetic factors.

Innovative technologies like array comparative genomic hybridization (arrayCGH), single nucleotide polymorphism (SNP) arrays and next-generation sequencing revealed recurrent mutations of genes implicated in epigenetic control such as ASXL1, EZH2, DNMT3A and TET2 in MDS and AML.

The human $A S X L 1$ gene is located on chromosome 20q11.21 and codes for part of the Polycombrepressive deubiquitinase complex, which functions to deubiquitylate H2AK119 [65]. EZH2 located on chromosome 7q36.1, is a histone methyltransferase that catalyzes trimethylation of $\mathrm{H} 3 \mathrm{~K} 27$ and is a subunit of the polycomb repressive complex 2 (PCR2). Polycomb proteins initiate and maintain transcriptional silencing through specific histone modifications. DNMT3A is a member of the mammalian family of methyltransferases that enzymatically add a methyl group to cytosine in CpG dinucleotides (cytosine and guanine separated by only one phosphate). Methylation of $\mathrm{CpG}$ sites within the promoters can lead to gene silencing, a frequent mechanism to inactivate tumor suppressor genes. Examples in MDS are $K L F 5, K L F 11$, and $M A F B$, shown to be aberrantly hypermethylated in $15 \%, 7 \%$, and $1.7 \%$ of 115 cases, respectively [66].

The Tet family was first identified as an oncofusion partner of the histone H3 Lys4 (H3K4) methyltransferase MLL in patients with the translocation $\mathrm{t}(10 ; 11)(\mathrm{q} 22 ; \mathrm{q} 23)$ in AML [67]. Recent

studies demonstrate that Tet proteins catalyze the conversion of 5-methylcytosine of DNA to 5-hydroxymethylcytosine $(5 \mathrm{hmC})$ and that TET2 mutations are associated with low $5 \mathrm{hmC}$ levels and 
global hypomethylation [68]. This suggests that an altered 5hmC status leads to deregulation of important hematopoietic regulators and contributes to malignancy [69].

\subsection{Role of Global DNA and Histone Methylation}

Novel techniques based on mass spectrometry allow large-scale quantitative DNA methylation analysis [32]. Using this approach, Bullinger et al. were able to report the first large-scale methylation-based outcome predictor in AML. Furthermore, they observed distinct methylation patterns for cytogenetic subgroups such as AML with $\operatorname{inv}(16), \mathrm{t}(8 ; 21), \mathrm{t}(15 ; 17)$ and $\mathrm{t}(11 \mathrm{q} 23)$. Also, microarray-based methods help to identify new clinical markers especially for AML with a normal karyotype and define new subgroups of AML. These techniques also allow the analysis of single promoter methylation patterns of specific genes. Promoter $\mathrm{CpG}$ methylation is often correlated with silencing of TSGs (tumor suppressor genes) in specific pathways that are also targets of mutation or other mechanisms of inactivation. Notably, TSGs silenced epigenetically often reside in genomic regions that are characterized by frequent chromosomal deletions. These results indicate that aberrant methylation can cooperate with chromosome deletions to silence TSG.

Using genome-wide approaches, unique methylation patterns of MDS and secondary AML were detected. Although particular cancer-related genes such as $C D K N 2 A$ and genes in the WNT signaling pathway were hypermethylated, epigenetic deregulation was not limited to cancer-associated genes but appeared to be a more widespread phenomenon [70].

Comparing low-risk MDS cases with a control group, 552 differentially methylated CpG loci were identified, while hypermethylated genes were more frequent than hypomethylated genes [71]. In another study, patients with higher levels of methylation, compared with patients with lower levels, had a shorter median overall survival (12.3 $v 17.5$ months, respectively) and shorter median progression-free survival (6.4 $v 14.9$ months, respectively) [72]. Shen et al. used these methylation analyses as a prognostic model and observed that this was independent of age, sex, and IPSS group.

So far, it is a matter of discussion whether mutation of single epigenetic regulators induce genetic instability. EVI1, a hematopoietic transcription factor that regulates stem cell renewal and induces epigenetic modifications [73], is frequently overexpressed in human myeloid neoplasia [74]. EVII rearrangement due to $\operatorname{inv}(3)$ or $\mathrm{t}(3 ; 3)$ is frequently associated with monosomy 7 and a very poor prognosis [75]. EVII is also a site of ectopic integration of retroviral vectors. Integration of a single retroviral vector into the EVII locus has led to leukemogenesis in mice [76,77]. Notably, in a human gene therapy trial, insertional mutagenesis was accompanied by the development of monosomy 7 . There are contradicting results as to whether EVI1 overexpression directly induces CIN via defective cytokinesis and accumulation of supernumerary centrosomes [78]. Thus, preleukemic dominance as a result of spontaneous chromosomal rearrangements or transgene integration events may be related to inhibited differentiation of hematopoietic stem cells [74].

Epigenetic modifications like DNA and histone methylation also play an important role in the induction of genomic instability. With the aim of studying the cooperation of genetic alterations and epigenetic modifications in the induction of genomic instability in leukemogenesis, we established a bone marrow transplantation model of myeloid leukemia in mice. The genetic defect resulted from an overexpression of the master oncogene $c-M y c$, whereas the epigenetic defect was induced by the 
histone methyltransferase Suv39h1 deficiency. Suv39h1 mediates the trimethylation of lysine 9 of histone 3 (H3K9me3) leading to condensed chromatin.

As expected, clonal chromosomal aberrations induced by $c-M y c$ overexpression were found in $c-M y c / w t$ leukemias of about one-third of primary recipients and in more than $80 \%$ of secondary recipients [79]. Notably, leukemias that arose in $c-M y c / S u v 39 h 1$-null mice were chromosomally stable, demonstrating that reduced H3K9 trimethylation due to lack of Suv39h1 rather protects leukemic cells from the development of CIN.

In our further analyses, we identified centromeric fusions with loss of telomeric signals and higher numbers of critically short telomeres in $c-M y c / S u v 39 h 1$-wt cells, but not in Suv39h1-null cells. Thus, telomere shortening to critical levels may have been prevented in Suv39h1-null cells, possibly due to better access of telomere-stabilizing proteins because of a more open chromatin structure. This is in accordance with the observation by Garcia-Cao et al. [80], who proved elongated telomeres in Suv39h1 and Suv39h2 double knock-out cells compared to wt cells. The critically short telomeres in leukemic $c-M y c / S u v 39 h 1$-wt cells may impair chromosomal stability by inducing breakage-fusion-bridge cycles resulting in e.g., centromeric fusions [81]. Furthermore, missegregation driven by telomere shortening may cause numerical chromosomal aberrations [82]. In our BM transplantation model, numerical chromosomal aberrations were found in primary recipients and centromeric fusions were found mostly in secondary recipients. This may indicate that progressive telomere shortening may be involved in clonal evolution, i.e., "mild" telomere shortening inducing chromosomal missegregation and numerical aberrations and more pronounced telomere shortening resulting in centromeric fusions and complex chromosomal rearrangements.

The heterogeneous telomere lengths and the detection of ALT-associated PML bodies (APBs) indicated that the ALT mechanism was mainly responsible for telomere maintenance in $c$-Myc-induced leukemias. Microarray analyses identified an increased expression of genes involved in the ALT mechanism such as Pml, Rad50, Smc5, Fen1, FancA, Mus81, Sp110 and Sp100 in c-Myc/Suv39h1-null leukemias. This is in agreement with the observation that knock-down of Rad50c and $S m c 5$ inhibits ALT-induced telomere maintenance, resulting in critically short telomeres [83]. Downregulation of Fen 1, FancA and Mus81 prevents telomeric recombination required for ALT [84]. The more effective elongation or stabilization of telomeres in $c-M y c$-driven myeloid leukemias by ALT mechanism may prevent telomere shortening and the induction of CIN in Suv39h1-deficient leukemias.

Consistent with studies by Karlsson et al. [85] and Ray et al. [86], we demonstrated a $c-M y c$-induced increase of DNA double-strand breaks (DSBs). However, DSBs were markedly reduced in $c-M y c /$ Suv39h1-null leukemias. Genes coding for repair proteins like Rad51c, Trp53bpl or Ccnf were upregulated in leukemic $c-M y c / S u v 39 h 1$-null cells. It has been shown that reduced expression of Rad51c, Trp53bpl or Ccnf is linked to chromosomal aberrations like sister chromatid fusions, aneuploidy or tetraploidy [87-89]. The up-regulation of these genes in leukemic c-Myc/Suv39h1-null cells may strengthen genomic stability due to improved DSB repair. Moreover, changes in the chromatin structure in Suv39h1-null cells may improve the accessibility of the DNA repair machinery. As shown by Goodarzi et al. [90], heterochromatin can be a barrier for physiological DSB repair and Suv39h1 and Suv39h2 double knock-out diminish DSB repair defects following Atm knock-out. Thus, highly efficient DSB repair might be a potential mechanism for preventing chromosomal aberrations in $c$ $M y c / S u v 39 h 1$-null leukemias. Our data showed for the first time that Suv39h1 deficiency may prevent 
genomic instability by more efficient DNA repair and telomere stabilization in hematopoietic bone marrow cells overexpressing $c-M y c$.

\section{Conclusions}

Even though there are many hints, the exact mechanism of chromosomal instability is still unclear. As we discuss in this review, telomere shortening and epigenetic deregulation may be involved in the development of chromosomal and genomic instability. Importantly, in leukemia, genomic instability and in particular CIN may lead to the evolution of a complex aberrant karyotype, which is associated with a poor prognosis. This raises the question whether early intervention in aberrant epigenetic processes or telomeric dysregulation may prevent the outgrowth of aberrant clones. Telomere length measurement may be integrated in risk assessment to predict the prognosis. Thus, telomeres and epigenetics most likely play an important role in the induction of chromosomal instability in cancer and particularly in myeloid neoplasia. Elucidation of the exact mechanism of CIN induction in myeloid neoplasia remains a promising aim to understand leukemogenesis and find new therapeutic approaches.

\section{Conflict of Interest}

The authors declare no conflict of interest.

\section{Acknowledgments}

We thank Gillian Teicke for preparation of the manuscript.

\section{References}

1. Cahill, D.P.; Kinzler, K.W.; Vogelstein, B.; Lengauer, C. Genetic instability and darwinian selection in tumours. Trends Cell. Biol. 1999, 9, M57-M60.

2. Nowak, M.A.; Komarova, N.L.; Sengupta, A.; Jallepalli, P.V.; Shih Ie, M.; Vogelstein, B.; Lengauer, C. The role of chromosomal instability in tumor initiation. Proc. Natl. Acad. Sci. USA 2002, 99, 16226-16231.

3. Lengauer, C.; Kinzler, K.W.; Vogelstein, B. Genetic instabilities in human cancers. Nature 1998, 396, 643-649.

4. Geigl, J.B.; Obenauf, A.C.; Schwarzbraun, T.; Speicher, M.R. Defining "chromosomal instability". Trends Genet. 2008, 24, 64-69.

5. Janssen, A.; Medema, R.H. Genetic instability: Tipping the balance. Oncogene 2012, doi:10.1038/onc.2012.576.

6. Bayani, J.; Selvarajah, S.; Maire, G.; Vukovic, B.; Al-Romaih, K.; Zielenska, M.; Squire, J.A. Genomic mechanisms and measurement of structural and numerical instability in cancer cells. Semin. Cancer Biol. 2007, 17, 5-18.

7. McGranahan, N.; Burrell, R.A.; Endesfelder, D.; Novelli, M.R.; Swanton, C. Cancer chromosomal instability: Therapeutic and diagnostic challenges. EMBO Rep. 2012, 13, 528-538. 
8. Wilkens, L.; Flemming, P.; Gebel, M.; Bleck, J.; Terkamp, C.; Wingen, L.; Kreipe, H.; Schlegelberger, B. Induction of aneuploidy by increasing chromosomal instability during dedifferentiation of hepatocellular carcinoma. Proc. Natl. Acad. Sci. USA 2004, 101, 1309-1314.

9. Heilig, C.E.; Loffler, H.; Mahlknecht, U.; Janssen, J.W.; Ho, A.D.; Jauch, A.; Kramer, A. Chromosomal instability correlates with poor outcome in patients with myelodysplastic syndromes irrespectively of the cytogenetic risk group. J. Cell Mol. Med. 2010, 14, 895-902.

10. Greenberg, P.L.; Tuechler, H.; Schanz, J.; Sanz, G.; Garcia-Manero, G.; Sole, F.; Bennett, J.M.; Bowen, D.; Fenaux, P.; Dreyfus, F.; et al. Revised international prognostic scoring system for myelodysplastic syndromes. Blood 2012, 120, 2454-2465.

11. Jadersten, M.; Hellstrom-Lindberg, E. New clues to the molecular pathogenesis of myelodysplastic syndromes. Exp. Cell. Res. 2010, 316, 1390-1396.

12. Swerdlow, S.; Campo, E.; Harris, N.; Jaffe, E.; Pileri, S.; Stein, H.; Thiele, J.; Vardiman, J. WHO Classification of Tumours of Haematopoietic and Lymphoid Tissues, 4th ed.; IARC: Lyon, France, 2008.

13. Malcovati, L.; Germing, U.; Kuendgen, A.; Della Porta, M.G.; Pascutto, C.; Invernizzi, R.; Giagounidis, A.; Hildebrandt, B.; Bernasconi, P.; Knipp, S.; et al. Time-dependent prognostic scoring system for predicting survival and leukemic evolution in myelodysplastic syndromes. J. Clin. Oncol. 2007, 25, 3503-3510.

14. Germing, U.; Strupp, C.; Giagounidis, A. Clinical features and prognosis of patients with myelodysplastic syndromes. Cancer Treat. Rev. 2007, 33, S15-S18.

15. Gohring, G.; Michalova, K.; Beverloo, H.B.; Betts, D.; Harbott, J.; Haas, O.A.; Kerndrup, G.; Sainati, L.; Bergstraesser, E.; Hasle, H.; et al. Complex karyotype newly defined: The strongest prognostic factor in advanced childhood myelodysplastic syndrome. Blood 2010, 116, 3766-3769.

16. Haase, D.; Germing, U.; Schanz, J.; Pfeilstocker, M.; Nosslinger, T.; Hildebrandt, B.; Kundgen, A.; Lubbert, M.; Kunzmann, R.; Giagounidis, A.A.; et al. New insights into the prognostic impact of the karyotype in MDS and correlation with subtypes: Evidence from a core dataset of 2124 patients. Blood 2007, 110, 4385-4395.

17. Pedersen-Bjergaard, J.; Andersen, M.T.; Andersen, M.K. Genetic pathways in the pathogenesis of therapy-related myelodysplasia and acute myeloid leukemia. Hematology Am. Soc. Hematol. Educ. Program 2007, 1, 392-397.

18. Corey, S.J.; Minden, M.D.; Barber, D.L.; Kantarjian, H.; Wang, J.C.; Schimmer, A.D. Myelodysplastic syndromes: The complexity of stem-cell diseases. Nat. Rev. Cancer 2007, 7, 118-129.

19. Huret, J.; Chomienne, C. t(15;17)(q24;q21). Available online: http://atlasgeneticsoncology.org/ Anomalies/t1517ID1035.html/ (accessed on 17 June 2013).

20. Dohner, H.; Estey, E.H.; Amadori, S.; Appelbaum, F.R.; Buchner, T.; Burnett, A.K.; Dombret, H.; Fenaux, P.; Grimwade, D.; Larson, R.A.; et al. Diagnosis and management of acute myeloid leukemia in adults: Recommendations from an international expert panel, on behalf of the European LeukemiaNet. Blood 2010, 115, 453-474. 
21. Lugthart, S.; Groschel, S.; Beverloo, H.B.; Kayser, S.; Valk, P.J.; van Zelderen-Bhola, S.L.; Jan Ossenkoppele, G.; Vellenga, E.; van den Berg-de Ruiter, E.; Schanz, U.; et al. Clinical, molecular, and prognostic significance of WHO type $\operatorname{inv}(3)(\mathrm{q} 21 \mathrm{q} 26.2) / \mathrm{t}(3 ; 3)(\mathrm{q} 21 ; \mathrm{q} 26.2)$ and various other 3q abnormalities in acute myeloid leukemia. J. Clin. Oncol. 2010, 28, 3890-3898.

22. Dohner, H.; Gaidzik, V.I. Impact of genetic features on treatment decisions in AML. Hematology Am. Soc. Hematol. Educ. Program 2011, 2011, 36-42.

23. Bejar, R.; Stevenson, K.; Abdel-Wahab, O.; Galili, N.; Nilsson, B.; Garcia-Manero, G.; Kantarjian, H.; Raza, A.; Levine, R.L.; Neuberg, D.; et al. Clinical effect of point mutations in myelodysplastic syndromes. N. Engl. J. Med. 2011, 364, 2496-2506.

24. Swiggers, S.J.; Kuijpers, M.A.; de Cort, M.J.; Beverloo, H.B.; Zijlmans, J.M. Critically short telomeres in acute myeloid leukemia with loss or gain of parts of chromosomes. Genes Chromosomes Cancer 2006, 45, 247-256.

25. Sieglova, Z.; Zilovcova, S.; Cermak, J.; Rihova, H.; Brezinova, D.; Dvorakova, R.; Markova, M.; Maaloufova, J.; Sajdova, J.; Brezinova, J.; et al. Dynamics of telomere erosion and its association with genome instability in myelodysplastic syndromes (MDS) and acute myelogenous leukemia arising from MDS: A marker of disease prognosis? Leuk. Res. 2004, 28, 1013-1021.

26. Boultwood, J.; Fidler, C.; Kusec, R.; Rack, K.; Elliott, P.J.; Atoyebi, O.; Chapman, R.; Oscier, D.G.; Wainscoat, J.S. Telomere length in myelodysplastic syndromes. Am. J. Hematol. 1997, 56, 266-271.

27. Blasco, M.A.; Lee, H.W.; Hande, M.P.; Samper, E.; Lansdorp, P.M.; DePinho, R.A.; Greider, C.W. Telomere shortening and tumor formation by mouse cells lacking telomerase RNA. Cell 1997, 91, 25-34.

28. Hemann, M.T.; Rudolph, K.L.; Strong, M.A.; DePinho, R.A.; Chin, L.; Greider, C.W. Telomere dysfunction triggers developmentally regulated germ cell apoptosis. Mol. Biol. Cell 2001, 12, 2023-2030.

29. Shay, J.W.; Wright, W.E. Senescence and immortalization: Role of telomeres and telomerase. Carcinogenesis 2005, 26, 867-874.

30. Bullinger, L.; Dohner, K.; Bair, E.; Frohling, S.; Schlenk, R.F.; Tibshirani, R.; Dohner, H.; Pollack, J.R. Use of gene-expression profiling to identify prognostic subclasses in adult acute myeloid leukemia. N. Engl. J. Med. 2004, 350, 1605-1616.

31. Alvarez, S.; Suela, J.; Valencia, A.; Fernandez, A.; Wunderlich, M.; Agirre, X.; Prosper, F.; Martin-Subero, J.I.; Maiques, A.; Acquadro, F.; et al. DNA methylation profiles and their relationship with cytogenetic status in adult acute myeloid leukemia. PLoS One 2010, 5, e12197.

32. Bullinger, L.; Ehrich, M.; Dohner, K.; Schlenk, R.F.; Dohner, H.; Nelson, M.R.; van den Boom, D. Quantitative DNA methylation predicts survival in adult acute myeloid leukemia. Blood 2010, $115,636-642$.

33. Nolte, F.; Giehl, M.; Haass, W.; Nowak, V.; Schumann, C.; Nowak, D.; Mossner, M.; Popp, H.D.; Schulze, T.J.; Klein, S.; et al. Centrosome aberrations in bone marrow cells from patients with myelodysplastic syndromes correlate with chromosomal instability. Ann. Hematol. 2013, doi:10.1007/s00277-013-1772-7.

34. Burrell, R.A.; McClelland, S.E.; Endesfelder, D.; Groth, P.; Weller, M.C.; Shaikh, N.; Domingo, E.; Kanu, N.; Dewhurst, S.M.; Gronroos, E.; et al. Replication stress links structural and numerical cancer chromosomal instability. Nature 2013, 494, 492-496. 
35. Rassool, F.V.; Gaymes, T.J.; Omidvar, N.; Brady, N.; Beurlet, S.; Pla, M.; Reboul, M.; Lea, N.; Chomienne, C.; Thomas, N.S.; et al. Reactive oxygen species, DNA damage, and error-prone repair: A model for genomic instability with progression in myeloid leukemia? Cancer. Res. 2007, 67, 8762-8771.

36. Sallmyr, A.; Tomkinson, A.E.; Rassool, F.V. Up-regulation of WRN and DNA ligase IIIalpha in chronic myeloid leukemia: Consequences for the repair of DNA double-strand breaks. Blood 2008, 112, 1413-1423.

37. Popp, H.D.; Bohlander, S.K. Genetic instability in inherited and sporadic leukemias. Genes Chromosomes Cancer 2010, 49, 1071-1081.

38. Drummond, M.W.; Balabanov, S.; Holyoake, T.L.; Brummendorf, T.H. Concise review: Telomere biology in normal and leukemic hematopoietic stem cells. Stem Cells 2007, 25, 1853-1861.

39. Hayflick, L. The limited in vitro lifetime of human diploid cell strains. Exp. Cell Res. 1965, 37, 614-636.

40. Hemann, M.T.; Strong, M.A.; Hao, L.Y.; Greider, C.W. The shortest telomere, not average telomere length, is critical for cell viability and chromosome stability. Cell 2001, 107, 67-77.

41. Calado, R.T.; Regal, J.A.; Hills, M.; Yewdell, W.T.; Dalmazzo, L.F.; Zago, M.A.; Lansdorp, P.M.; Hogge, D.; Chanock, S.J.; Estey, E.H.; et al. Constitutional hypomorphic telomerase mutations in patients with acute myeloid leukemia. Proc. Natl. Acad. Sci. USA 2009, 106, 1187-1192.

42. Shay, J.W.; Wright, W.E. Telomeres and telomerase in normal and cancer stem cells. FEBS Lett. 2010, 584, 3819-3825.

43. Martinez, P.; Blasco, M.A. Telomeric and extra-telomeric roles for telomerase and the telomere-binding proteins. Nat. Rev. Cancer 2011, 11, 161-176.

44. Blackburn, E.H.; Collins, K. Telomerase: An RNP enzyme synthesizes DNA. Cold Spring Harb. Perspect. Biol. 2011, doi:10.1101/chsperspect.a003558.

45. Artandi, S.E. Telomeres, telomerase, and human disease. N. Engl. J. Med. 2006, 355, 1195-1197.

46. Drummond, M.W.; Hoare, S.F.; Monaghan, A.; Graham, S.M.; Alcorn, M.J.; Keith, W.N.; Holyoake, T.L. Dysregulated expression of the major telomerase components in leukaemic stem cells. Leukemia 2005, 19, 381-389.

47. Murnane, J.P. Telomere loss as a mechanism for chromosome instability in human cancer. Cancer Res. 2010, 70, 4255-4259.

48. Hande, M.P. DNA repair factors and telomere-chromosome integrity in mammalian cells. Cytogenet. Genome Res. 2004, 104, 116-122.

49. Gisselsson, D. Mitotic instability in cancer: Is there method in the madness? Cell Cycle 2005, 4, 1007-1010.

50. Briatore, F.; Barrera, G.; Pizzimenti, S.; Toaldo, C.; Casa, C.D.; Laurora, S.; Pettazzoni, P.; Dianzani, M.U.; Ferrero, D. Increase of telomerase activity and hTERT expression in myelodysplastic syndromes. Cancer Biol. Ther. 2009, 8, 883-889.

51. Pettigrew, K.A.; Armstrong, R.N.; Colyer, H.A.; Zhang, S.D.; Rea, I.M.; Jones, R.E.; Baird, D.M.; Mills, K.I. Differential TERT promoter methylation and response to 5-aza-2'deoxycytidine in acute myeloid leukemia cell lines: TERT expression, telomerase activity, telomere length, and cell death. Genes Chromosomes Cancer 2012, 51, 768-780. 
52. Rollison, D.E.; Epling-Burnette, P.K.; Park, J.Y.; Lee, J.H.; Park, H.; Jonathan, K.; Cole, A.L.; Painter, J.S.; Guerrier, M.; Melendez-Santiago, J.; et al. Telomere length in myelodysplastic syndromes. Leuk. Lymphoma 2011, 52, 1528-1536.

53. Capraro, V.; Zane, L.; Poncet, D.; Perol, D.; Galia, P.; Preudhomme, C.; Bonnefoy-Berard, N.; Gilson, E.; Thomas, X.; El-Hamri, M.; et al. Telomere deregulations possess cytogenetic, phenotype, and prognostic specificities in acute leukemias. Exp. Hematol. 2011, 39, 195-202.e2.

54. Gadji, M.; Adebayo Awe, J.; Rodrigues, P.; Kumar, R.; Houston, D.S.; Klewes, L.; Dieye, T.N.; Rego, E.M.; Passetto, R.F.; de Oliveira, F.M.; et al. Profiling three-dimensional nuclear telomeric architecture of myelodysplastic syndromes and acute myeloid leukemia defines patient subgroups. Clin. Cancer Res. 2012, 18, 3293-3304.

55. Calado, R.T.; Young, N.S. Telomere maintenance and human bone marrow failure. Blood 2008, $111,4446-4455$.

56. Young, N.S. Telomere biology and telomere diseases: Implications for practice and research. Hematology Am. Soc. Hematol. Educ. Program 2010, 2010, 30-35.

57. Armanios, M. Syndromes of telomere shortening. Annu. Rev. Genomics Hum. Genet. 2009, 10, 45-61.

58. Kirwan, M.; Vulliamy, T.; Marrone, A.; Walne, A.J.; Beswick, R.; Hillmen, P.; Kelly, R.; Stewart, A.; Bowen, D.; Schonland, S.O.; et al. Defining the pathogenic role of telomerase mutations in myelodysplastic syndrome and acute myeloid leukemia. Hum. Mutat. 2009, 30, 1567-1573.

59. Calado, R.T.; Cooper, J.N.; Padilla-Nash, H.M.; Sloand, E.M.; Wu, C.O.; Scheinberg, P.; Ried, T.; Young, N.S. Short telomeres result in chromosomal instability in hematopoietic cells and precede malignant evolution in human aplastic anemia. Leukemia 2012, 26, 700-707.

60. Yamaguchi, H.; Baerlocher, G.M.; Lansdorp, P.M.; Chanock, S.J.; Nunez, O.; Sloand, E.; Young, N.S. Mutations of the human telomerase RNA gene (TERC) in aplastic anemia and myelodysplastic syndrome. Blood 2003, 102, 916-918.

61. Lange, K.; Holm, L.; Vang Nielsen, K.; Hahn, A.; Hofmann, W.; Kreipe, H.; Schlegelberger, B.; Gohring, G. Telomere shortening and chromosomal instability in myelodysplastic syndromes. Genes Chromosomes Cancer 2010, 49, 260-269.

62. Gohring, G.; Lange, K.; Hofmann, W.; Nielsen, K.V.; Hellstrom-Lindberg, E.; Roy, L.; Morgan, M.; Kreipe, H.; Busche, G.; Giagounidis, A.; et al. Telomere shortening, clonal evolution and disease progression in myelodysplastic syndrome patients with $5 \mathrm{q}$ deletion treated with lenalidomide. Leukemia 2012, 26, 356-358.

63. Sobulo, O.M.; Borrow, J.; Tomek, R.; Reshmi, S.; Harden, A.; Schlegelberger, B.; Housman, D.; Doggett, N.A.; Rowley, J.D.; Zeleznik-Le, N.J. MLL is fused to CBP, a histone acetyltransferase, in therapy-related acute myeloid leukemia with a t(11;16)(q23;p13.3). Proc. Natl. Acad. Sci. USA 1997, 94, 8732-8737.

64. Katsumoto, T.; Yoshida, N.; Kitabayashi, I. Roles of the histone acetyltransferase monocytic leukemia zinc finger protein in normal and malignant hematopoiesis. Cancer Sci. 2008, 99, 1523-1527. 
65. Scheuermann, J.C.; de Ayala Alonso, A.G.; Oktaba, K.; Ly-Hartig, N.; McGinty, R.K.; Fraterman, S.; Wilm, M.; Muir, T.W.; Muller, J. Histone H2A deubiquitinase activity of the Polycomb repressive complex PR-DUB. Nature 2010, 465, 243-247.

66. Potapova, A.; Hasemeier, B.; Romermann, D.; Metzig, K.; Gohring, G.; Schlegelberger, B.; Langer, F.; Kreipe, H.; Lehmann, U. Epigenetic inactivation of tumour suppressor gene KLF11 in myelodysplastic syndromes. Eur. J. Haematol. 2010, 84, 298-303.

67. Ono, R.; Taki, T.; Taketani, T.; Taniwaki, M.; Kobayashi, H.; Hayashi, Y. LCX, leukemiaassociated protein with a CXXC domain, is fused to MLL in acute myeloid leukemia with trilineage dysplasia having t(10;11)(q22;q23). Cancer Res. 2002, 62, 4075-4080.

68. Koh, K.P.; Yabuuchi, A.; Rao, S.; Huang, Y.; Cunniff, K.; Nardone, J.; Laiho, A.; Tahiliani, M.; Sommer, C.A.; Mostoslavsky, G.; et al. Tet1 and Tet2 regulate 5-hydroxymethylcytosine production and cell lineage specification in mouse embryonic stem cells. Cell Stem Cell 2011, 8, 200-213.

69. Matarese, F.; Carrillo-de Santa Pau, E.; Stunnenberg, H.G. 5-hydroxymethylcytosine: A new kid on the epigenetic block? Mol. Syst. Biol. 2011, 7, 562.

70. Figueroa, M.E.; Skrabanek, L.; Li, Y.; Jiemjit, A.; Fandy, T.E.; Paietta, E.; Fernandez, H.; Tallman, M.S.; Greally, J.M.; Carraway, H.; et al. MDS and secondary AML display unique patterns and abundance of aberrant DNA methylation. Blood 2009, 114, 3448-3458.

71. del Rey, M.; O’Hagan, K.; Dellett, M.; Aibar, S.; Colyer, H.A.; Alonso, M.E.; Diez-Campelo, M.; Armstrong, R.N.; Sharpe, D.J.; Gutierrez, N.C.; et al. Genome-wide profiling of methylation identifies novel targets with aberrant hypermethylation and reduced expression in low-risk myelodysplastic syndromes. Leukemia 2013, 27, 610-618.

72. Shen, Y.; Zhu, Y.M.; Fan, X.; Shi, J.Y.; Wang, Q.R.; Yan, X.J.; Gu, Z.H.; Wang, Y.Y.; Chen, B.; Jiang, C.L.; et al. Gene mutation patterns and their prognostic impact in a cohort of 1185 patients with acute myeloid leukemia. Blood 2011, 118, 5593-5603.

73. Lugthart, S.; Figueroa, M.E.; Bindels, E.; Skrabanek, L.; Valk, P.J.; Li, Y.; Meyer, S.; Erpelinck-Verschueren, C.; Greally, J.; Lowenberg, B.; et al. Aberrant DNA hypermethylation signature in acute myeloid leukemia directed by EVI1. Blood 2011, 117, 234-241.

74. Kustikova, O.S.; Schwarzer, A.; Stahlhut, M.; Brugman, M.H.; Neumann, T.; Yang, M.; Li, Z.; Schambach, A.; Heinz, N.; Gerdes, S.; et al. Activation of Evil inhibits cell cycle progression and differentiation of hematopoietic progenitor cells. Leukemia 2012, 27, 1127-1138.

75. Lugthart, S.; van Drunen, E.; van Norden, Y.; van Hoven, A.; Erpelinck, C.A.; Valk, P.J.; Beverloo, H.B.; Lowenberg, B.; Delwel, R. High EVI1 levels predict adverse outcome in acute myeloid leukemia: Prevalence of EVI1 overexpression and chromosome 3q26 abnormalities underestimated. Blood 2008, 111, 4329-4337.

76. Li, Z.; Dullmann, J.; Schiedlmeier, B.; Schmidt, M.; von Kalle, C.; Meyer, J.; Forster, M.; Stocking, C.; Wahlers, A.; Frank, O.; et al. Murine leukemia induced by retroviral gene marking. Science 2002, 296, 497.

77. Modlich, U.; Schambach, A.; Brugman, M.H.; Wicke, D.C.; Knoess, S.; Li, Z.; Maetzig, T.; Rudolph, C.; Schlegelberger, B.; Baum, C. Leukemia induction after a single retroviral vector insertion in Evil or Prdm16. Leukemia 2008, 22, 1519-1528. 
78. Karakaya, K.; Herbst, F.; Ball, C.; Glimm, H.; Kramer, A.; Loffler, H. Overexpression of EVI1 interferes with cytokinesis and leads to accumulation of cells with supernumerary centrosomes in G0/1 phase. Cell Cycle 2012, 11, 3492-3503.

79. Vajen, B.; Modlich, U.; Schienke, A.; Wolf, S.; Skawran, B.; Hofmann, W.; Busche, G.; Kreipe, H.; Baum, C.; Santos-Barriopedro, I.; et al. Histone methyltransferase Suv39h1 deficiency prevents Myc-induced chromosomal instability in murine myeloid leukemias. Genes Chromosomes Cancer 2013, 52, 423-430.

80. Garcia-Cao, M.; O’Sullivan, R.; Peters, A.H.; Jenuwein, T.; Blasco, M.A. Epigenetic regulation of telomere length in mammalian cells by the Suv39h1 and Suv39h2 histone methyltransferases. Nat. Genet. 2004, 36, 94-99.

81. Louis, S.F.; Vermolen, B.J.; Garini, Y.; Young, I.T.; Guffei, A.; Lichtensztejn, Z.; Kuttler, F.; Chuang, T.C.; Moshir, S.; Mougey, V.; et al. c-Myc induces chromosomal rearrangements through telomere and chromosome remodeling in the interphase nucleus. Proc. Natl. Acad. Sci. USA 2005, 102, 9613-9618.

82. Pampalona, J.; Soler, D.; Genesca, A.; Tusell, L. Whole chromosome loss is promoted by telomere dysfunction in primary cells. Genes Chromosomes Cancer 2010, 49, 368-378.

83. Zhong, Z.H.; Jiang, W.Q.; Cesare, A.J.; Neumann, A.A.; Wadhwa, R.; Reddel, R.R. Disruption of telomere maintenance by depletion of the MRE11/RAD50/NBS1 complex in cells that use alternative lengthening of telomeres. J. Biol. Chem. 2007, 282, 29314-29322.

84. Fan, Q.; Zhang, F.; Barrett, B.; Ren, K.; Andreassen, P.R. A role for monoubiquitinated FANCD2 at telomeres in ALT cells. Nucleic Acids Res. 2009, 37, 1740-1754.

85. Karlsson, A.; Deb-Basu, D.; Cherry, A.; Turner, S.; Ford, J.; Felsher, D.W. Defective double-strand DNA break repair and chromosomal translocations by MYC overexpression. Proc. Natl. Acad. Sci. USA 2003, 100, 9974-9979.

86. Ray, S.; Atkuri, K.R.; Deb-Basu, D.; Adler, A.S.; Chang, H.Y.; Herzenberg, L.A.; Felsher, D.W. MYC can induce DNA breaks in vivo and in vitro independent of reactive oxygen species. Cancer Res. 2006, 66, 6598-6605.

87. Smeenk, G.; de Groot, A.J.; Romeijn, R.J.; van Buul, P.P.; Zdzienicka, M.Z.; Mullenders, L.H.; Pastink, A.; Godthelp, B.C. Rad51C is essential for embryonic development and haploinsufficiency causes increased DNA damage sensitivity and genomic instability. Mutat. Res. 2010, 689, 50-58.

88. Fung, T.K.; Siu, W.Y.; Yam, C.H.; Lau, A.; Poon, R.Y. Cyclin F is degraded during G2-M by mechanisms fundamentally different from other cyclins. J. Biol. Chem. 2002, 277, 35140-35149.

89. Ward, I.M.; Minn, K.; van Deursen, J.; Chen, J. p53 binding protein 53BP1 is required for DNA damage responses and tumor suppression in mice. Mol. Cell. Biol. 2003, 23, 2556-2563.

90. Goodarzi, A.A.; Noon, A.T.; Jeggo, P.A. The impact of heterochromatin on DSB repair. Biochem. Soc. Trans. 2009, 37, 569-576.

(C) 2013 by the authors; licensee MDPI, Basel, Switzerland. This article is an open access article distributed under the terms and conditions of the Creative Commons Attribution license (http://creativecommons.org/licenses/by/3.0/). 